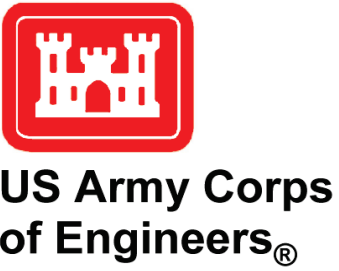

\title{
Basic Logic for the Construction of an AdH Estuarine Model
}

by Jennifer McAlpin and Jeremy Sharp

PURPOSE: The purpose of this Coastal and Hydraulics Laboratory (CHL) technical note is to present the engineering procedure for building models to investigate estuarine processes using the two-dimensional (2D) shallow water equations in the Adaptive Hydraulics (AdH) finite element code.

INTRODUCTION: For this document, the term model implies the conceptual assumptions, userspecified parameters, and the mesh definition utilized in the $\mathrm{AdH}$ code. There are no numerical code modifications to AdH discussed herein.

Estuarine modeling presents unique challenges that require forethought and perception to both construct the model (conceptual assumptions) and understand numerical code limitations. The need for forethought in assumptions and parameterization in setting up applications is intrinsic to all hydraulic modeling. Engineering experience through exposure to a broad range of estuarine projects is the best instructor for construction of estuarine models. Often, especially for novice engineers, the temptation is to over value model results without the proper application and understanding of model limitations. The constructed model is only a representation of the prototype and is not infallible. Careful consideration is required to provide the greatest probability of successful interpretation of field behavior with a numerical model.

Below is a step-by-step thought logic for users to understand the important processes and considerations when constructing an estuarine model that correctly represents the desired domain. The following sections include discussion on defining the domain, boundary conditions, validation considerations, and model result interpretation. This instructional technical note assumes the user has previous exposure to $\mathrm{AdH}$; thus, this is supplemental information intended to aid the user and not cover the nuances of applying the code. For a full understanding of the code, it is recommended to review the AdH User Manual (CHL 2017) and participate in AdH training.

MODEL INITIATION: The model initiation process starts by properly defining the modeling objective and collecting critical data. Typical objectives for 2D estuarine modeling include analysis of tidal exchange, tidal wetting and drying, sedimentation, salinity intrusion, mixing, roughness changes, flood protection structures, navigation channels, etc. Often the analysis is conducted for pre- and post-project conditions where comparisons are made between the two. The project objective must conform to the limitations of the $\mathrm{AdH} 2 \mathrm{D}$ code. For example, near field flow around a complex hydraulic structure is a three-dimensional (3D) problem; thus, 2D assumptions are invalid. However, analysis of the distance associated with energy loss from a structure may fit within the limitations of the AdH 2D code. Salinity intrusion is a 3D process; however, AdH includes several corrections to model 3D processes with the $2 \mathrm{D}$ code. Understanding the assumptions and the project intent is critical to knowing if the $\mathrm{AdH} 2 \mathrm{D}$ code is applicable. A way of evaluating the applicability is determining the dominant processes (i.e., horizontal eddies can be represented in the $2 \mathrm{D}$ code, but 
vertical eddies cannot). Additionally, the objective must be fully understood such that the model is constructed to properly provide a solution. One common mistake is to evaluate behavior in the model near the boundary. Here, the flow conditions may be influenced by the boundary specification and not allowed to properly develop resulting in erroneous values. Thus, the inclusion of additional domain area would be required.

The proper data, in type and amount (spatially and temporally), are required to accomplish the modeling objective. Often field data must be acquired to attain the required data for model construction and validation. Prior to any study, data should be thoroughly mined in all available resources. The necessary data include bathymetry/topography, aerial georeferenced images, bed roughness or land use type, hydrodynamics, both discharge and water surface elevation, and data pertaining to the location of man-made features (dikes, revetments, bridges, dams, grade control, etc.). Once these data are compiled and properly georeferenced, model construction can initiate as defined in the following sections and further expounded in the provided example.

DEFINING THE DOMAIN: The domain physically encompasses the geometry necessary to properly define the study site. Study sites for estuaries are typically areas where changes are required to address navigation, flood control, or environmental issues. The study site area and the region around it must be defined (mesh resolution and details) to correctly represent the bathymetry and the hydraulics. To achieve this, one must view the geometry in all three dimensions. In the horizontal (X and Y) plane, the domain is defined by the upstream (bay boundaries and river inflows) and downstream (open ocean or water body) cross sections and the side boundaries or tidal extent. Then, in the vertical $(Z)$, the domain is defined with the bed geography/bathymetric and topographical data. Understanding that this is a $2 \mathrm{D}$ model is critical; thus, the horizontal $\mathrm{X}$ and $\mathrm{Y}$ directions are being solved, and certain assumptions (beyond the scope of this discussion) are being made about the vertical, $\mathrm{Z}$ (i.e., hydrostatic pressure).

Fundamentally, site geometry dictates flow conditions/patterns. Thus, the domain extents must be sufficient to capture the area that defines the flow - such as the tidal propagation and extent of tidal influence. Defining/determining the domain limits of the study site is directly related and dependent on the desired interest of the user. The user must know what is being studied to define the domain limits. In practical applications, most user interests lie in the use of the model to determine impacts from potential changes in the field, for example the impact of closing an inlet on the salinity intrusion into an estuary. Answering this question requires an understanding of the site and how the simulated flows might react to the changes. As explained previously, near field flow conditions, such as near structures (jetties), will not be appropriately captured with the 2D AdH code. For example, the vertical velocities at a jetty will not be captured, so scour potential of the structures cannot be evaluated. This limitation indicates that the user should use extreme caution when applying the calculated velocities and depths near the structures. However, the far field effects, away from structures, can be appropriately interpreted. The area of structural influence will vary based on site-specific characteristics. When defining the user interest for the model, local structural impacts should be avoided, but away from those same structures the model has the ability to behave correctly (i.e., the salinity changes in the estuary, and general $2 \mathrm{D}$ velocity patterns can be modeled accurately).

Once the site of interest has been defined, the extent of tidal influence of the estuary must also be understood. This distance can be determined by observing gage data through the estuary and into 
the rivers/creeks that enter the estuary. The model should extend to a point where the tidal signal is no longer observed in the water surface elevation. If this extent is not possible, such as at the location of a dam or flow control structure, appropriate boundary conditions can be applied at this location, but the study area should be far enough from this boundary such that the flow is allowed to fully establish prior to the period of interest.

The mesh resolution of the domain must be defined to adequately represent the geometry, bathymetry, and hydraulic conditions. Flow channels should have a minimum of three elements across the width to allow for adequate conveyance of the flow. AdH 2D can dynamically refine the mesh for hydraulic and transport conditions based on user-supplied parameters, but the initial mesh must be adequate to represent the domain geometry and bathymetry and to visualize model results.

BOUNDARY CONDITIONS: The boundary conditions define the spatial and temporal inputs for the model. In most estuarine applications of $\mathrm{AdH}$, the upstream boundary (riverine input) is specified with a total discharge, and the offshore boundary (open water) is specified with a water surface elevation, often referred to as a tail water elevation. At the downstream tidal boundary, it is often necessary to specify more than one tail water elevation and boundary condition due to the variation in water surface elevation over the distance of the boundary. There are multiple approaches that can be taken to determine how to best define the tidal boundary, many dependent on the location and extents of this boundary. The boundary can be specified using measured data or harmonic constituents. The boundary conditions can be constant along the length of the boundary or vary based on known data. Most important, however, is to reproduce the speed of the tide wave and the timing of the flow, especially when there are multiple entrances into an estuary. The boundary definition is also important in reproducing larger offshore circulation patterns. Additional boundary conditions include bed roughness parameters, eddy viscosity, constituent diffusion, and mesh adaption parameters. The necessary input parameters are dependent on the specific model application.

VALIDATION: The comparison of a model's performance to what is observed in the field is known as validation. Fundamentally, validation means to solve the right equations such that the model is physically relevant to the field (Wang et al. 2009). The success of the validation process is achieved through the adjustment of conceptual assumptions (i.e., calibration). Adjustments to model parameters that specify the conceptual assumptions should always be physically realistic. For example, Manning's bed roughness coefficient, $n$, is only valid up to certain depths (see the Friction Control section of the AdH User Manual; CHL [2017]).

For the AdH code, as related to estuarine modeling of hydrodynamics and salinity, the primary adjustment parameters are bed roughness, and to a limited extent eddy viscosity and salinity diffusion. Roughness is often specified in either values of Manning's $n$ or roughness height. The recommended range for the correct application of Manning's $n$ is $0.01-0.04$. Desired roughness outside this range should be specified with roughness height. Roughness height is $2-3$ times the D90 (90 percent finer of sediment grain size) of the bed gradation. When vegetation is present, the unsubmerged and/or submerged vegetation roughness options should be utilized. Secondary controls are eddy viscosity and salinity diffusion. Eddy viscosity is dictated by the flow depth and velocity of the system. Salinity diffusion includes the spreading of the constituent due to vertical variations in velocity and Brownian diffusion of particles. These values should remain as low as possible although limited tweaking is permissible. Additional guidance should be sought in the 
AdH User Manual (CHL 2017). When making adjustments to the model, it is recommended to make one change per model run. If multiple changes are made simultaneously, then it is impossible to determine the impact of each modification.

In most cases, data for model validation are often limited. However, the modeler can usually get water surface elevation data from National Oceanic and Atmospheric Administration (NOAA), U.S. Geological Survey, or a local agency. Water surface elevation is a good place to start with model validation. Current or velocity data and/or cross-sectional discharge are necessary to ensure the direction of the tidal flow and the associated tidal prism is accurate. Salinity measurements for validation are often more difficult to obtain but are typically collected by local agencies monitoring water quality. Acceptable errors in the validation of the model are based on the uncertainty of the input data and the specific intent of the project and are therefore evaluated on a case-by-case basis. Often tracking the propagation of the tide wave into the estuary is a good way to determine where energy is being lost and if model parameter changes are necessary. If the tide propagation is correct in the open water and then diverges from the field measurements closer inland, the geometry (including bathymetry) or bed roughness parameters should be examined. The mesh resolution may be inadequate to capture the geometry/bathymetry or to pass the necessary flow, especially if part of the channel is dry.

A mesh convergence test is recommended when validating a model to determine if enough resolution is included. The $\mathrm{AdH}$ adaptive meshing feature helps in performing this test. To conduct a mesh convergence test in $\mathrm{AdH}$, refine the base mesh globally or include the adaption feature in $\mathrm{AdH}$ and compare the model results for the two meshes. If the results change significantly, perform the test again (increase adaption levels and/or lower adaption tolerances). Repeat this process until the results no longer vary between the finest mesh and the prior one. Figure 1 shows the meshes and results for a mesh convergence test of a concentration plume being advected from left to right down a simple flume. The concentrations depicted in the third and fourth images are very similar, indicating that using the third mesh (2525 nodes) provides an adequately converged solution.

Most estuarine modeling is simulated over several tidal cycles. It is recommended that the model be validated to a range of flow conditions that can be accomplished by making multiple shortduration simulations or by simulating an extended hydrograph (12-18 months). Salinity requires many tidal cycles to initialize; thus, an additional 3-6 months of simulation is often performed as an initialization, or spin-up, for the model. Velocity and water surface elevations typically initialize in 1-2 tidal cycles.

Validation is often based on error metrics that combine the time history of the simulation comparisons into a single value for a given parameter and location, such as root mean square (RMS) error (perfect is 0.0) and correlation coefficient (perfect is 1.0). These metrics are useful, but there are instances where looking at plots of the comparisons over time is of great value. Box plots showing the field and model results on separate axes are useful to determine the spread in the model validation and identify consistent phase and/or amplitude errors. Figure 2 shows a timeseries comparison on the left and a box-plot comparison on the right using the same data. The RMS error and the correlation coefficient are also provided. A model's ability to accurately represent the field is directly related to the user's ability to supply correct input conditions and validate the model. 


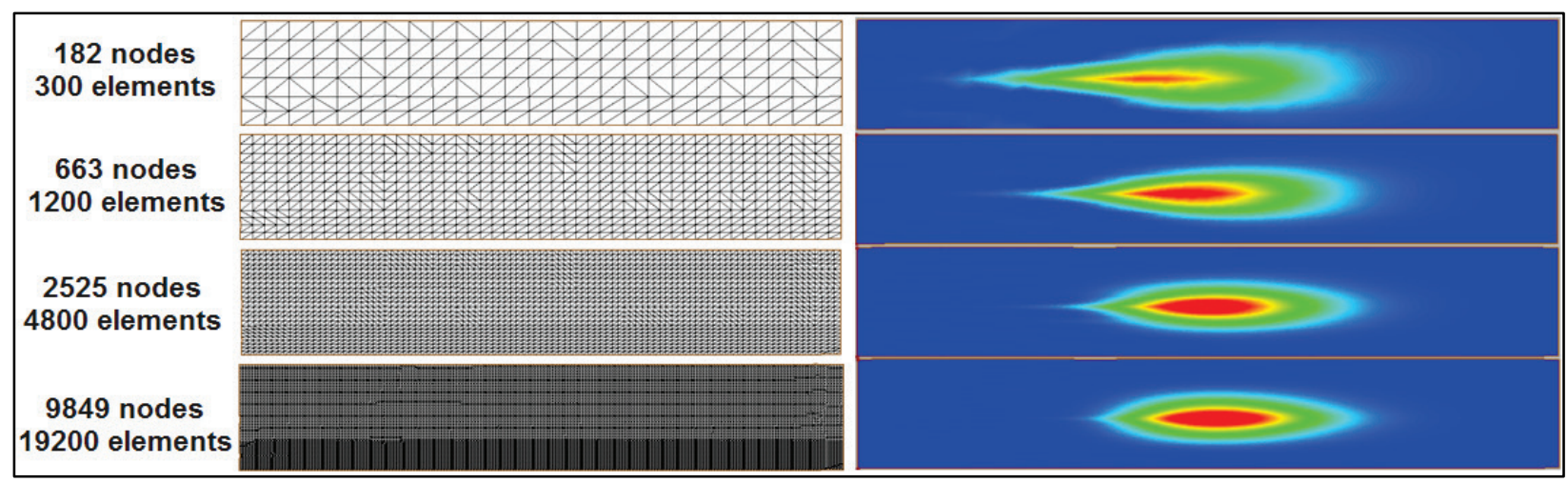

Figure 1. Mesh convergence test.
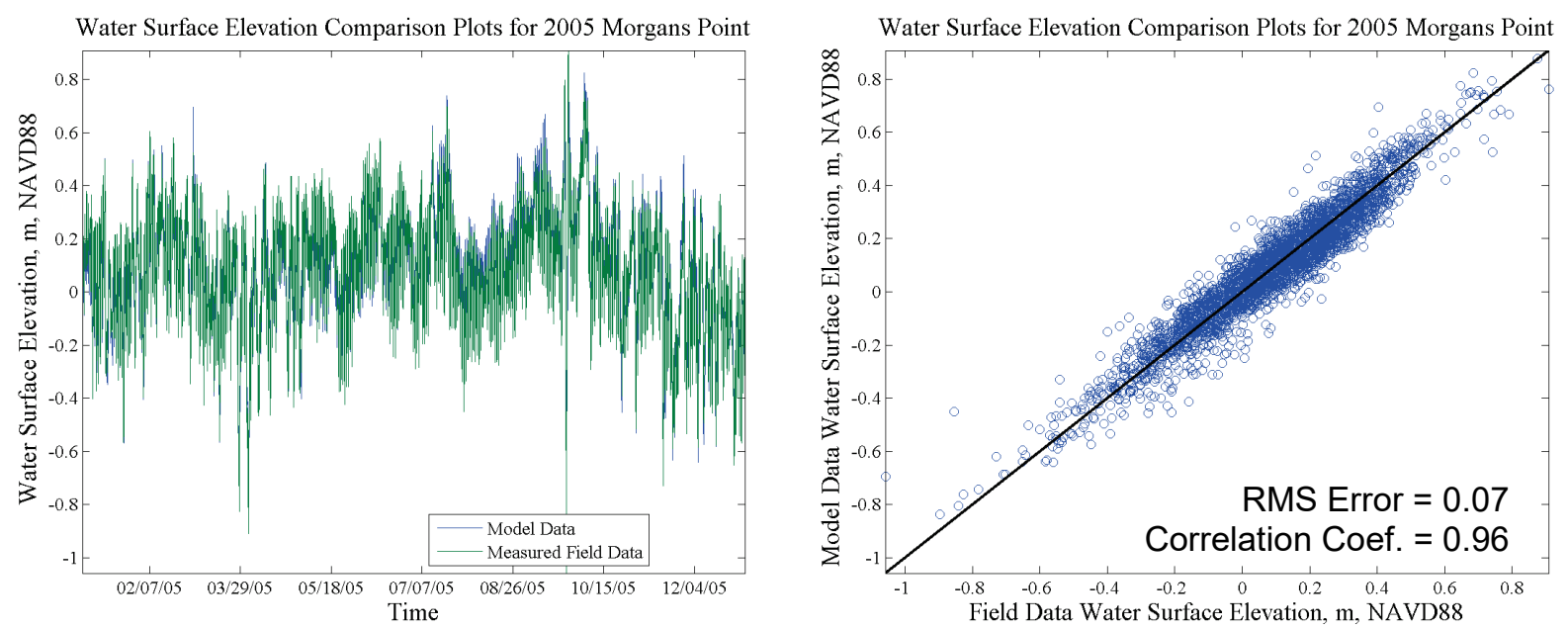

Figure 2. Model/field comparison plots (left: time series, right: box).

INTERPRETATION: All model results, including those from estuarine models, require engineering judgment for correct interpretation. An understanding of the code and system are required to quantitatively evaluate the model solution. The user must understand that a model is merely a representation of nature. There is a level of cynicism that is beneficial when reviewing results. Always ask why, what, and how the model and code are representing the domain. Modelers may be tempted to overvalue the results and believe whatever the output might show.

For the purpose of understanding the model as it represents the domain, it is suggested that model parameter sensitivity testing always be performed. Model parameter sensitivity is performed by varying the conceptual assumptions and seeing how the variations change the solution. The flow, tailwater, wind, roughness, eddy viscosity, bed slope or geometric features could all be altered to grasp how the model might respond. If the response seems consistent, then the maximum and minimum of the sensitivity results might be applied to all model results to bound the results or show the uncertainty of the model results. However, if the response is contrary or inconsistent, then there is a high probability that the model is not converging on the correct solution or there is a fundamental error in the model construction or assumptions. 
COMPARISION: The primary purpose for estuarine modeling is usually to understand how a system might respond to proposed changes. Changes typically involve the construction or modification of features such as navigation channels, dredge placement sites, and flood control structures. Since these features are proposed and do not exist in the field, validation is done for the existing conditions. Once validated, the model is only modified to reflect the proposed conditions. Both the existing and proposed condition results can then be analyzed to determine the impact of the proposed changes.

EXAMPLE: To illustrate the above logic, an example is outlined here. The example uses the Mississippi River Gulf Outlet (MRGO), Gulf Intercoastal Waterway, and Lake Pontchartrain system (Tate et al. 2010), and the Surface Water Modeling System (SMS) for mesh generation and visualization (for tutorials, see $\underline{w w w . a q u a v e o . c o m}$ ). These steps illustrate the construction of an estuarine hydrodynamic model:

1. Understanding and appropriately defining the horizontal and vertical control, so all input files used to construct the model are geo-referenced with the same projections. This is imperative when working in general but is extremely important when processing several files. Additionally, geo-referencing ensures the ability to bring in files after initial model testing, thus providing a living model that can evolve with the study. For this example, the project is defined in North American Vertical Datum of 1988 (2004.65 epoch), feet, and North American Datum of 1983 State Plane Coordinate System, Louisiana South, feet.

2. Start delineating the SMS map file with a correctly geo-referenced aerial image, which includes the area of interest including the anticipated/required domain limits. The image is useful in the delineation of bank lines, vegetation boundaries, overbank limits, levee locations, structures, etc. Figure 3 shows an overview of the study area with key details denoted.

3. Structures such as bridge piers, jetties, flood walls, revetments, grade-control, dikes, weirs, and levees are best located with a shape or computer-aided design file. It is critical to locate these correctly for delineation in the SMS map file. Hard features control the geometry and geometry dictates the hydrodynamics.

4. Node spacing on feature arcs in the map module of SMS should be geometrically consistent. It is best to limit node spacing changes for adjacent feature arcs such that the transition from one arc to the next is not too large. This spacing ultimately defines the mesh resolution; thus, careful consideration of mesh requirements for various features and visualization are necessary at this point. Figure 4 (left) shows the map arcs and polygons that will be used to generate the mesh. 


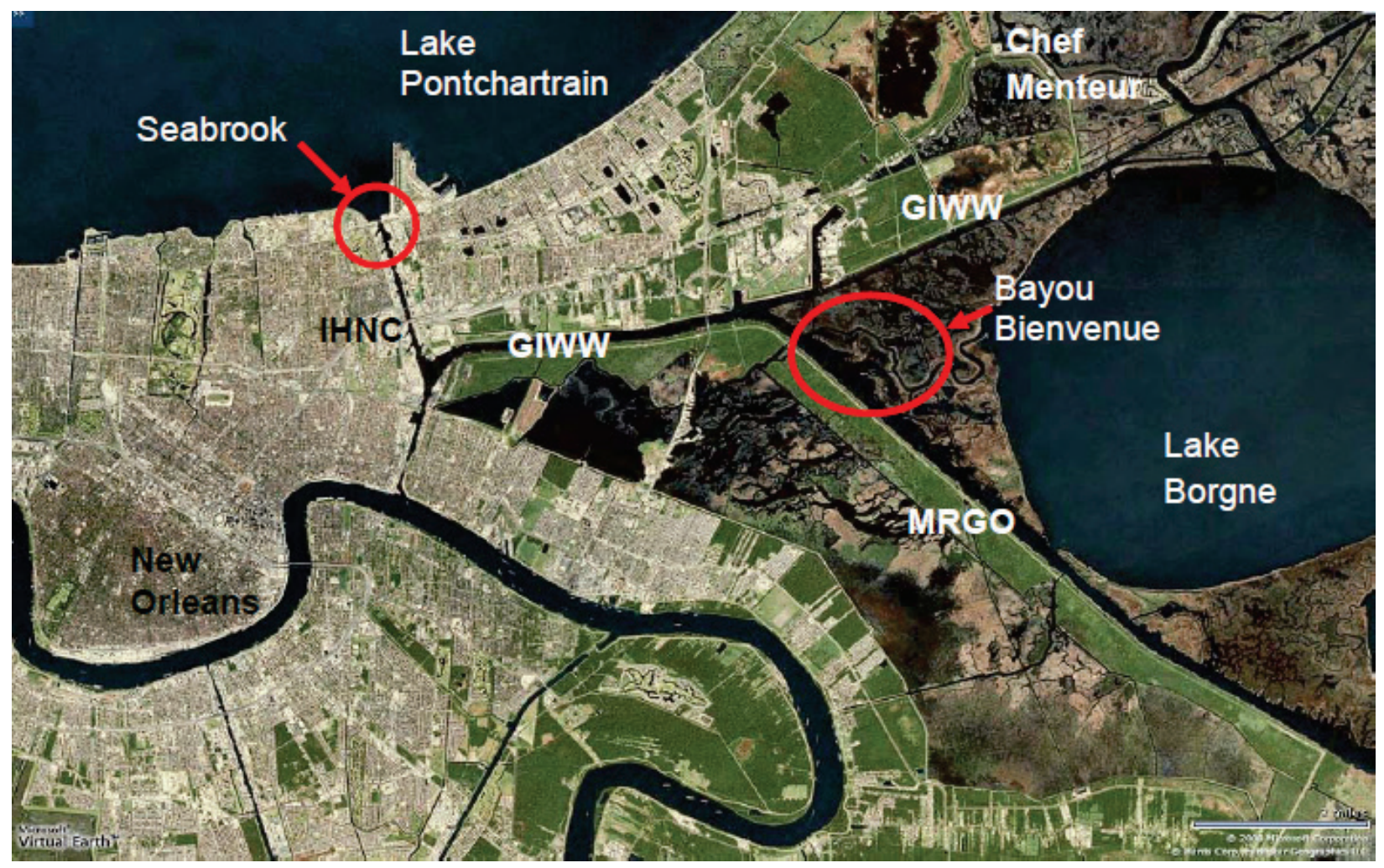

Figure 3. Aerial image of demonstration site (New Orleans, LA).

5. Once all necessary features are correctly located in the domain with appropriate node spacing, the mesh can then be generated (Figure 4, right). The map features are converted to polygons, and the roughness values are assigned. At this point, the scatter bathymetry data can be included in the polygon properties or later incorporated into the mesh. It is critical to create enough mesh resolution to capture both the geometric features and hydrodynamic processes as the mesh resolution dictates the model scale. Eddies or other flow phenomena of a smaller scale than the mesh resolution will not be captured in the modeling.

6. Scatter sets define the bathymetry/topography of the domain. It is important to ensure the scatter set sufficiently defines the bathymetry. Key features should be captured in the data and are required to establish the correct flow patterns. Figure 5 shows color-shaded contours of the bathymetry that has been interpolated to the mesh from a scatter data set.

7. Verify that the mesh resolution captures the hydraulic and geometric features and that the contours match the location of these features. The left image in Figure 6 shows the mesh and elevation contours as defined around bridge piers and sector gate structures. The right image shows the MRGO entering into the Gulf of Mexico. The bathymetric interpolation should match with the added resolution of the MRGO channel. If the interpolation scheme is incorrect or the coordinates do not match, the scatter data will not interpolate accurately onto the mesh resulting in an improper representation of the system. 


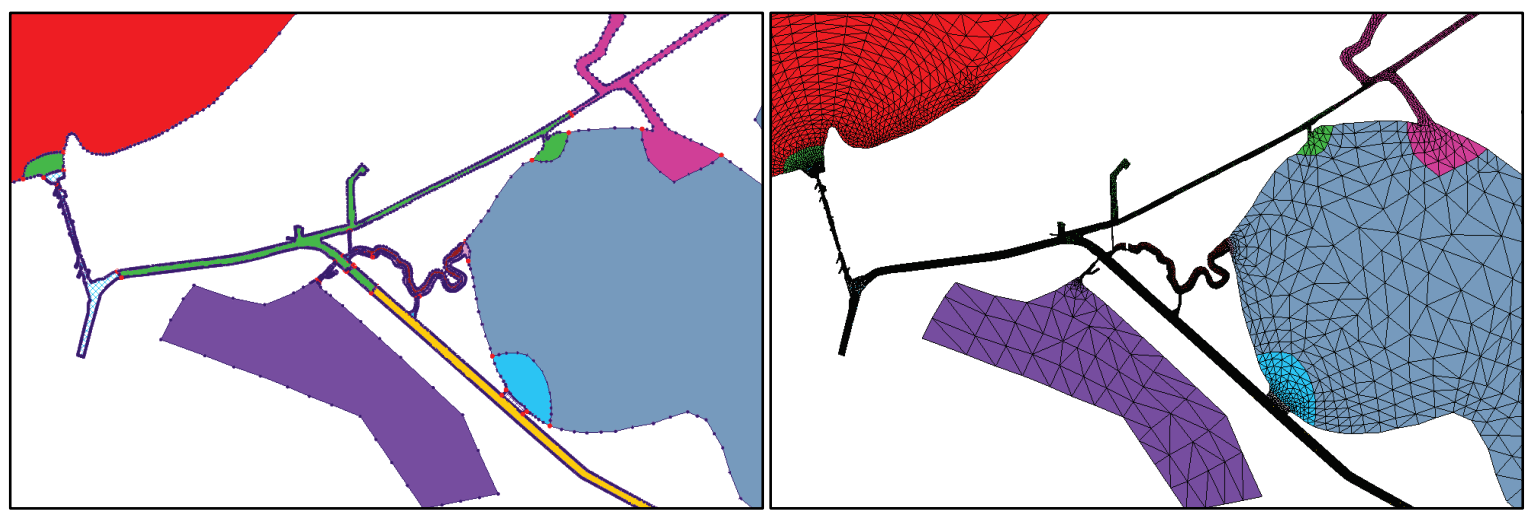

Figure 4. Map arcs, vertex spacing and material definitions (left), with mesh (right).

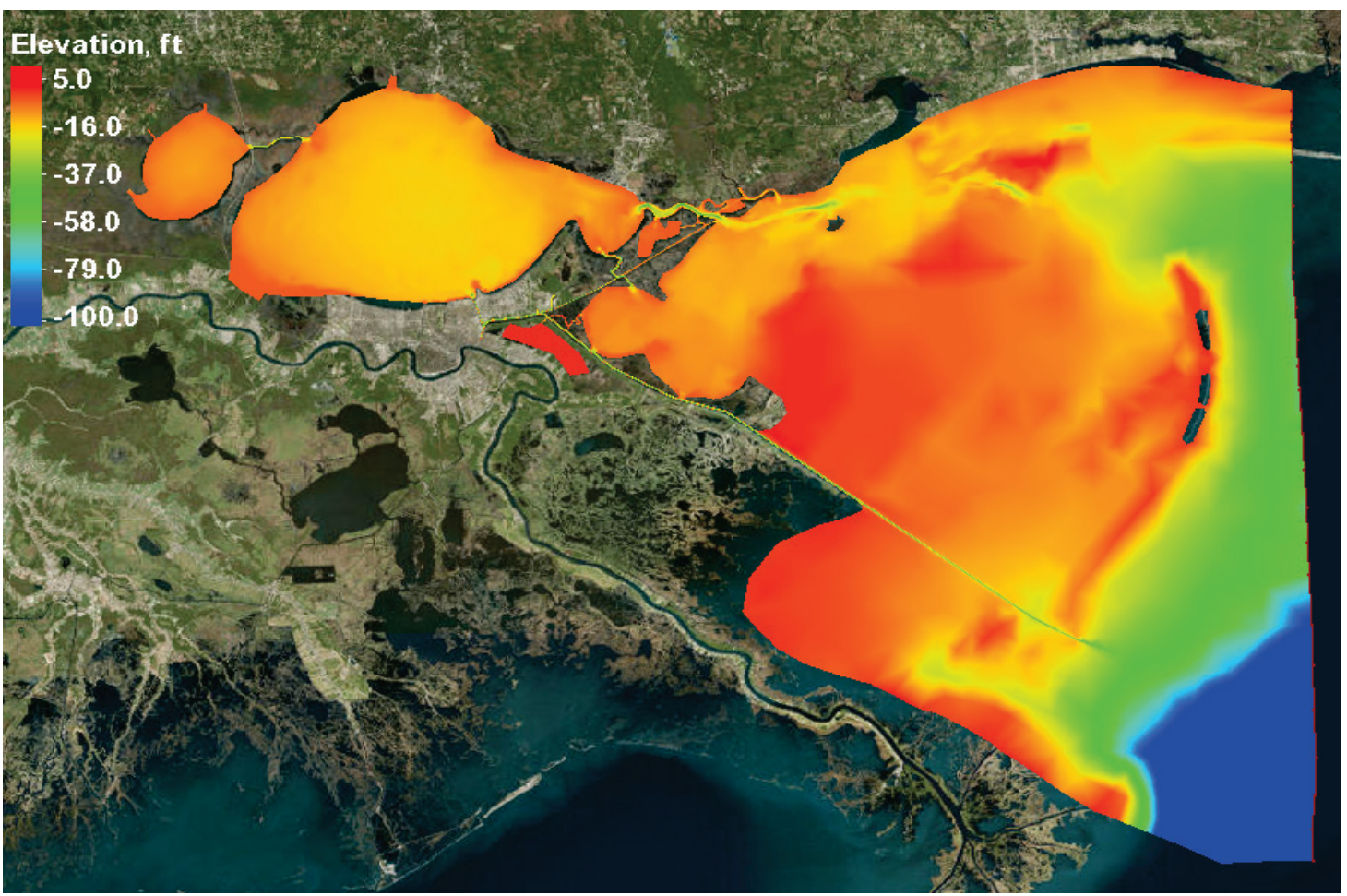

Figure 5. Mesh with bathymetry interpolated.

8. The input, or boundary condition (bc), file sets the parameters of the model for the code. This model has a tail water boundary condition on the eastern boundary at the Gulf of Mexico. The water surface elevation at this location is interpolated from NOAA gages at Gulfport Harbor and Pilots Station East, Southwest Pass. River inflows are supplied as a time history of total discharge for six rivers that enter the model domain. Surface wind data are specified using data available at the New Orleans International Airport. Wind data are often filtered to remove highfrequency noise; removing signals less than 3 hours in duration provides a more accurate and numerically stable signal. Engineering judgment must be used when filling gaps in a data set. Missing data should be filled based on interpolation for short gaps in time or by applying data from nearby gages for longer data gaps. Reference the AdH Users Manual for details on generating the boundary condition file (CHL 2017). 

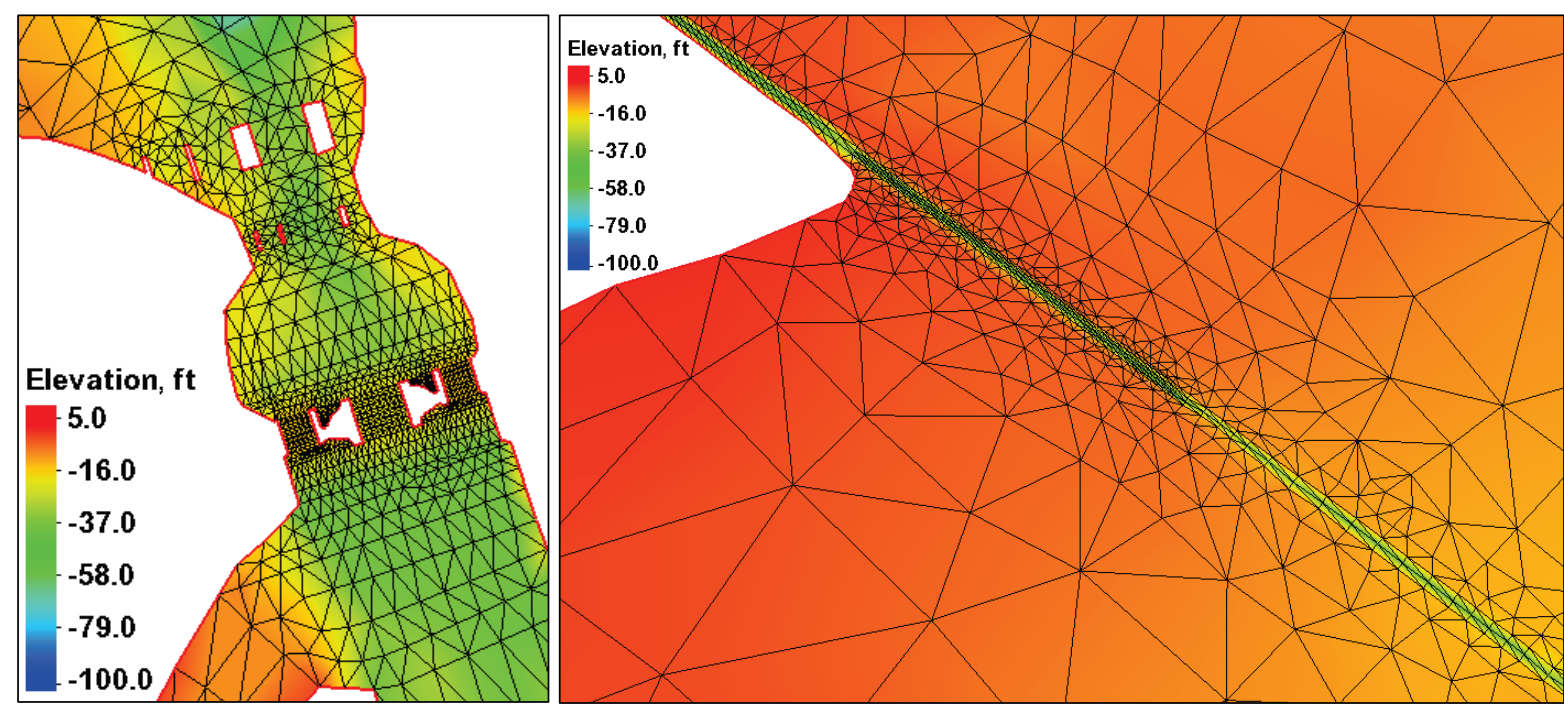

Figure 6. Verify the mesh resolution and contours match the features as intended.

9. The initial conditions, or hot start (hot), file for a model provides the code with the correct initial depths and velocities (if appropriate). All AdH models are required to begin with water depths in this file. This model is set up to have a flat water surface elevation at the start, so the sum of the water depth and the bed elevation equals a constant water surface elevation at all mesh nodes. The value is set to match the initial tide elevation at the boundary to provide a smooth starting condition for the model. Discontinuities between the initial conditions and the initial boundary conditions can result in model instabilities.

10. Once all three input files (mesh, bc, and hotstart) are created, model simulations for without project conditions (existing conditions or base) can be performed. This model is simulated on the U.S. Army Engineer Research and Development Center (ERDC) High Performance Computing machines to perform multi-processor simulations. General AdH guidance suggests approximately 1,000 nodes per processor but varies based on applications. This mesh contains just under 20,000 nodes, so 20 processors are a reasonable number of processors for acceptable run performance. Timing tests on various processor counts can be performed to optimize the simulation efficiency.

11. Validation can be performed on the existing conditions by processing the model output for the specific comparisons needed. AdH produces model output at a user-specified interval. Estuarine modeling is tidally driven, so comparisons should be performed throughout a tidal cycle. It is recommended to save model results at least every 3-4 hours with increased output required for highly dynamic locations. Figure 2 depicts comparisons to water surface elevation, velocity, and discharge for this model.

12. Once the model is proven to match field conditions within an acceptable level of accuracy, the model can be used to perform simulations with modified input conditions and/or geometries. However, it is important to always maintain an understanding of the assumptions made when developing and validating the model so that it is being used within those limitations. Comparisons to alternative conditions that violate the limitations of the model will not result in accurate base versus plan comparisons and could result in misleading results. 
13. Model sensitivity tests can be performed by adjusting certain model parameters within a reasonable range and observing how the results change from the base condition. Typically, sensitivity tests are performed on input parameters that are not measured, such as bed roughness or viscosity/mixing terms or with measured values that are known to have measurement uncertainty (flow, tailwaters). The variation in the hydrodynamic response provides a potential range of impacts associated with the adjusted parameter.

14. From the model simulations, engineers can make informed decisions on the behavior of a proposed feature. These decisions can include modifications to the proposed changes, further testing, code modifications (to address shortcomings in the code's ability to correctly model the study site), and recommendations or limitations to simulated features, alternatives, and much more.

CONCLUSION: Models are useful tools that can inform decision making. Like all tools, they must be appropriately applied and interpreted. Thus, understanding the model limitations and using a systematic approach is imperative for proper execution. Here, a systematic approach and logic has been presented, but it is not infallible nor all encompassing. Depending on the project and its goals, modifications to the process presented here may be required.

ADDITIONAL INFORMATION: This technical note was prepared by Jennifer McAlpin and Jeremy A. Sharp, Research Hydraulic Engineers at the ERDC Coastal and Hydraulics Laboratory. This effort was funded through the U.S. Army Corps of Engineers Numerical Model Maintenance Program. Questions about this technical note can be addressed to Ms. McAlpin at Jennifer.N.McAlpin@usace.army.mil.

This CHETN should be referenced as follows:

McAlpin, J., and J. Sharp. 2019. Basic Logic for the Construction of an AdH Estuarine Model. ERDC/CHL CHETN-IV-117. Vicksburg, MS: U.S. Army Engineer Research and Development Center. $\underline{\text { http://dx.doi.org/10.21079/11681/32543 }}$

\section{REFERENCES}

Coastal and Hydraulics Laboratory. 2017. Adaptive Hydraulics 2D Shallow Water (AdH-SW2D) User Manual (Version 4.6). Vicksburg, MS: U.S. Army Engineer Research and Development Center. https://chl.erdc.dren.mil/chladh

Tate, J. N., T. C. Lackey, and T. O. McAlpin. 2010. Seabrook Fish Larval Transport Study. ERDC/CHL TR10-12. Vicksburg, MS: U.S. Army Engineer Research and Development Center.

Wang, S. S., P. J. Roache, Y. Jia, R. A. Schmalz, and P. E. Smith, eds. 2009. Verification and Validation of ${ }_{3} D$ Free-Surface Flow Models. American Society of Civil Engineers. https://doi.org/10.1061/9780784409572.cho1

NOTE: The contents of this technical note are not to be used for advertising, publication or promotional purposes. Citation of trade names does not constitute an official 